\title{
Studying stars by going underground: the LUNA experiment at Gran Sasso Laboratory
}

\author{
Alessandra Guglielmetti for the LUNA collaboration \\ Università degli Studi di Milano and INFN Sezione di Milano, Via Celoria 16, I-20133 Milano, Italy
}

\begin{abstract}
Cross sections of astrophysically relevant reactions are essential ingredients for element nucleosynthesis, energy generation and neutrino fluxes in stellar environments. The low value of such cross sections in the stellar energy range prevents their measurement in a laboratory at the Earth's surface where the signal to background ratio would be too small. The LUNA collaboration has exploited the unique features of background reduction offered by the rock overburden above the LNGS underground laboratory in order to study very important Hydrogen-burning reactions. Future perspectives include the installation of a MV machine underground to be able to study also Helium burning reactions.
\end{abstract}

\section{Introduction}

Nuclear astrophysics is an extremely rich field strongly correlated with many other research fields like observational astronomy, neutrino physics, cosmology, nuclear physics etc. Since stars are powered by nuclear reactions, their cross sections are an essential ingredient of stellar models and many of the presently available data do not have the required precision.

Thermonuclear fusion reactions in stars start from the most abundant and lightest element, hydrogen, and gradually synthesize heavier elements [1]. The first nuclear fusion process is the socalled Hydrogen burning which has the net result of transforming 4 protons into a ${ }^{4} \mathrm{He}$ nucleus with an energy release of about $27 \mathrm{MeV}$. Hydrogen burning coincides with the longest stage of a star's life (also known as its main sequence phase) and is responsible for the prodigious luminosity of the star itself. It mainly proceeds either through the p-p chain or through the more efficient CNO cycle. The latter and successive cycles such as the $\mathrm{NeNa}$ and $\mathrm{MgAl}$ become important for second generation stars whose central temperatures and masses are higher than those of our Sun and whose evolution stage is such that the necessary seeds for these reactions are already present. Due to the higher Coulomb barriers, these cycles are relatively unimportant for energy generation but are essential for the nucleosynthesis of elements with mass number higher than 20.

All these fusion reactions occur in a very well defined energy range, the so-called Gamow peak [1], which arises from the convolution of the energy distribution of nuclei in the stellar plasma and the tunnelling probability through the Coulomb barrier between the interacting nuclei. In a nondegenerate, non-relativistic stellar plasma, the former is given by the Maxwell-Boltzmann distribution, with a maximum for $\mathrm{E}=\mathrm{kT}$ (where $\mathrm{T}$ is the star temperature and $\mathrm{k}$ the Maxwell-Boltzmann constant) and then decreases exponentially with increasing energy, while the latter decreases exponentially for decreasing energy. For example, for a central temperature of $1.510^{7} \mathrm{~K}$, as in our Sun, the maximum 
of the Maxwell-Boltzmann distribution occurs at about $1 \mathrm{keV}$, while the Coulomb barrier for most reactions in either the p-p chain or the $\mathrm{CNO}$ cycle is between 0.5 and $2 \mathrm{MeV}$. The Gamow peak for the same reactions is below $30 \mathrm{keV}$ and, as a result, reaction cross sections can be extremely low, down to the femto-barn level, due to the already mentioned exponential drop of the tunnelling probability with decreasing energy. It follows that a direct investigation of thermonuclear reactions at or near their Gamow energy is often beyond technical capabilities as the signal-to-noise ratio is severely dominated by any source of unwanted background. In a laboratory at the Earth's surface, the greatest contribution to the background typically arises from the interaction of cosmic rays with the material in the detection setup. Reaction cross-sections are therefore extrapolated from data taken at higher energies, albeit with significant uncertainties remaining at the lowest energies of astrophysical interest. The extrapolation procedure is typically carried out on the so-called astrophysical S-factor defined by:

$$
S(E)=\mathrm{E} \sigma(E) \exp (2 \pi \eta)
$$

where $\eta$ is the Sommerfeld parameter given by:

$$
\eta=(31.29 / 2 \pi) Z_{1} Z_{2}(\mu / E)^{1 / 2}
$$

with $Z_{1}$ and $Z_{2}$ integral charges of the interacting nuclei; $\mu$ the reduced mass; and $E$ the centre-of-mass interaction energy in $\mathrm{keV}$. The astrophysical $\mathrm{S}$-factor has a much reduced energy dependence compared to the cross section. However, the existence of narrow resonances in or near the Gamow energy region, the tails of broad resonances and/or sub-threshold states, as well as a change in the reaction mechanism at ultra-low energies, can all translate in non-negligible contributions to the reaction cross section (or equivalently the astrophysical factor) thus rendering the extrapolation procedure extremely uncertain.

An alternative solution to the extrapolation procedure requires a drastic reduction of any unwanted background so as to optimise the signal-to-noise ratio. This can often be achieved by carrying out the measurement in deep underground laboratories.

\section{The LUNA project in the underground Gran Sasso Laboratory}

The LUNA (Laboratory for Underground Nuclear Astrophysics) collaboration has exploited the lowbackground environment of the underground laboratory under the Gran Sasso Mountain in Italy (LNGS) to perform direct measurements at the relevant astrophysical energies. The rock overburden of about $1400 \mathrm{~m}$ (3800 m water equivalent) reduces the muon component of the cosmic background by a factor of $10^{6}$; the neutron component by a factor of $10^{3}$; and the gamma component by a factor of 10 with respect to a laboratory on the Earth's surface. As a result, the gamma background above 3 $\mathrm{MeV}$ in an HPGe detector placed underground at LNGS is reduced by a factor of $\sim 2500$ with respect to the same detector placed over-ground. In addition, going underground enhances the effect of passive shielding particularly for lower energy gammas where the background is dominated by environmental radioactivity. Indeed, a passive shield can be built around the detector also in a laboratory at the Earth's surface. However, passive shielding are of limited utility since additional background from interactions of the cosmic rays with the shielding material overwhelms the positive effect of attenuation in the shielding itself. An exception is the extremely large thickness which can be achieved in an underground laboratory, where the cosmic background is much reduced.

The LUNA collaboration has installed two accelerators underground: a compact $50 \mathrm{kV}$ "homemade" machine [2] and a commercial $400 \mathrm{kV}$ one [3]. Common features of the two are the intense beam currents achievable, the long-term stability, and the precise energy determination. The first two 
features are essential to maximize the reaction rate, while the third one is important in view of the exponential energy dependence of the cross section. With the first machine, operating between 1992 and 2001, two key reactions of the p-p chain were studied at the solar Gamow peak energies: the ${ }^{3} \mathrm{He}\left({ }^{3} \mathrm{He}, 2 \mathrm{p}\right){ }^{4} \mathrm{He}[4]$ and $\mathrm{d}(\mathrm{p}, \gamma)^{3} \mathrm{He}$ [5]. In addition, the screening effect, i.e. lowering of the Coulomb barrier for a nucleus surrounded by electrons with respect to a bare one, was investigated through the $\mathrm{d}\left({ }^{3} \mathrm{He}, \mathrm{p}\right)^{4} \mathrm{He}$ reaction [6]. The $400 \mathrm{kV}$ machine started operations in the year 2000 and is still operating. The most important results obtained include the study of the ${ }^{14} \mathrm{~N}(\mathrm{p}, \gamma){ }^{15} \mathrm{O}$ reaction $[7,8,9]$, the slowest reaction of the $\mathrm{CNO}$ cycle governing its energy and neutrino production. These results have important consequences on the CNO neutrino flux in the Sun, almost proportional to the Sfactor, which is reduced of a factor of about 2. Moreover, the ${ }^{14} \mathrm{~N}(\mathrm{p}, \gamma){ }^{15} \mathrm{O}$ reaction in core hydrogen burning affects the age of globular clusters, i.e. conglomerates of $10^{4}$ to $10^{6}$ gravitationally bound stars among the oldest stars in our galaxy. The LUNA measurements allowed to increase the age of globular clusters by 0.7 to 1 billion years [10], thus raising the lower limit on the age of the Universe up to 14 billion years. Another important measurement concerned the ${ }^{3} \mathrm{He}\left({ }^{4} \mathrm{He}, \gamma\right){ }^{7} \mathrm{Be}$ reaction: Solar ${ }^{7} \mathrm{Be}$ and ${ }^{8} \mathrm{~B}$ neutrinos depend almost linearly on the cross section of such reaction. Moreover, the ${ }^{3} \mathrm{He}\left({ }^{4} \mathrm{He}, \gamma\right){ }^{7} \mathrm{Be}$ reaction plays a critical role in the Big-Bang nucleosynthesis $(\mathrm{BBN})$, being the main source of ${ }^{7} \mathrm{Li}$ production. The LUNA result $[11,12,13]$ was included, together with other high precision measurements, in the recent compilation "Solar fusion II" [14] to derive the best astrophysical S-factor for the ${ }^{3} \mathrm{He}\left({ }^{4} \mathrm{He}, \gamma\right){ }^{7} \mathrm{Be}$ reaction. The value obtained allows for an uncertainty reduction in the solar neutrino fluxes from $7.5 \%$ to $4.3 \%$ in the case of ${ }^{8} \mathrm{~B}$ and from $8 \%$ to $4.5 \%$ in the case of ${ }^{7} \mathrm{Be}[15]$. Other key reactions belonging to the $\mathrm{CNO}\left({ }^{15} \mathrm{~N}(\mathrm{p}, \gamma){ }^{16} \mathrm{O}[16]\right.$ and ${ }^{17} \mathrm{O}(\mathrm{p}, \gamma){ }^{18} \mathrm{~F}[17]$ and contribution by A. Caciolli in these Proceedings) or $\operatorname{MgAl}\left({ }^{25} \operatorname{Mg}(\mathrm{p}, \gamma)^{26} \mathrm{Al}[18,19,20]\right)$ cycles of Hydrogen burning have also been measured with the $400 \mathrm{kV}$ machine while others are presently under data analysis $\left({ }^{2} \mathrm{H}(\alpha, \gamma){ }^{6} \mathrm{Li}\right.$ [see C. Gustavino in these Proceedings]) or data taking $\left({ }^{17} \mathrm{O}(\mathrm{p}, \alpha){ }^{14} \mathrm{~N}\right.$ and ${ }^{22} \mathrm{Ne}(\mathrm{p}, \gamma){ }^{23} \mathrm{Na}$ [see F. Cavanna in these Proceedings]). Most important LUNA results are included in the two quite recent reviews [21, 22].

\section{The future and the LUNA- MV project}

After the completion of the above cited measurements, the LUNA $400 \mathrm{kV}$ machine will be used to study a few more $(p, \gamma)$ and $(p, \alpha)$ reactions on Oxygen and Sodium isotopes. All of them can be studied with the $400 \mathrm{kV}$ accelerator since the relevant energy range, or at least part of it, is accessible to such a machine. After that, reactions that take place at higher temperatures (i.e. higher energies) than those occurring during the hydrogen-burning processes studied so far are planned to be measured using a higher voltage machine, in the MV range. For this reason, already in 2007, the LUNA collaboration submitted a Letter of Intent (LoI) [23] to the LNGS Scientific Committee which contained an experimental program mainly devoted to Helium burning reactions. In particular the ${ }^{12} \mathrm{C}(\alpha, \gamma){ }^{16} \mathrm{O}$ and the $(\alpha, \mathrm{n})$ reactions on ${ }^{13} \mathrm{C}$ and ${ }^{22} \mathrm{Ne}$ were foreseen.

The cross section of the ${ }^{12} \mathrm{C}(\alpha, \gamma){ }^{16} \mathrm{O}$ reaction at the relevant Gamow energy $(\sim 300 \mathrm{keV})$ determines the helium burning time scale and, together with the convection mechanism, the abundances of carbon and oxygen at the end of helium burning. The carbon abundance at the end of helium burning has important consequences for the subsequent evolution of the star and for the nucleosynthesis path. In particular, the amount of intermediate-light elements $\mathrm{Ne}, \mathrm{Na}, \mathrm{Mg}$, and $\mathrm{Al}$ ejected during a type II Supernova explosion scales directly with this parameter, while the amount of the elements produced in the advanced explosive burning phases beyond carbon burning scale inversely [24]. The situation is similar for type I Supernovae. Thus, stellar models show an exceptional sensitivity on the cross section of ${ }^{12} \mathrm{C}(\alpha, \gamma){ }^{16} \mathrm{O}$ and an experimental determination with a precision of the order of $10 \%$ or better is required to provide adequate constraints on stellar evolution. A measurement at the astrophysical energy is unfeasible due to the extremely low cross section and the astrophysical reaction rate relies on an extrapolation of existing data. This extrapolation of high-energy data is 
complicated by a complex reaction scheme. The lack of low-energy data with high precision implies that significant progress on the determination of the ${ }^{12} \mathrm{C}(\alpha, \gamma){ }^{16} \mathrm{O}$ cross section can only be made by an extensive, dedicated project in a low background environment with the primary focus on measurements of the ground state transition through angular distribution. The experiment will strongly benefit from the low background environment at higher $\gamma$-ray energies in the LNGS.

The ${ }^{13} \mathrm{C}(\alpha, n){ }^{16} \mathrm{O}$ and the ${ }^{22} \mathrm{Ne}(\alpha, \mathrm{n}){ }^{25} \mathrm{Mg}$ are the so-called $\mathrm{n}$-source reactions as they are responsible of the n-flux for the creation of the heavy elements. Indeed, more than 50 years ago Burbidge, Burbidge, Fowler \& Hoyle [25] proposed neutron-captures as the path to build up most elements heavier than iron. Since then, much effort was spent to understand the neutron-capture nucleosynthesis in stars of different mass and composition. Major neutron-capture processes are the sprocess and r-process. While little is known about astrophysical sites where the r-process takes place, the basic properties of the s-process have been identified. Two different components of the s-process can be distinguished: (i) the weak component, responsible for all isotopes with masses $60<\mathrm{A}<90$, is supposed to take place during the hydrostatic evolution of massive stars; (ii) the main component, responsible for heavier isotopes (from $\mathrm{Kr}$ to $\mathrm{Bi}$ ), occurs in Asymptotic Giant Branch stars. The major sources for the required neutron flux are the ${ }^{13} \mathrm{C}(\alpha, \mathrm{n}){ }^{16} \mathrm{O}$ and ${ }^{22} \mathrm{Ne}(\alpha, \mathrm{n}){ }^{25} \mathrm{Mg}$ reactions that operate under different conditions: the former has a positive Q-value $(2.216 \mathrm{MeV})$ while the latter has a negative one of $-0.478 \mathrm{MeV}$ and it can only be associated with higher temperature conditions. In lowmass AGB stars the ${ }^{13} \mathrm{C}(\alpha, n){ }^{16} \mathrm{O}$ reaction is the major neutron source. The precise experimental study of its reaction rate with related uncertainty is fundamental for the distribution of the s-process elements in the stellar surface material. Therefore, a direct measurement in the relevant temperature range is mandatory and will increase our knowledge on the heavy element production. The study of the ${ }^{13} \mathrm{C}(\alpha, n){ }^{16} \mathrm{O}$ reaction at astrophysical energies requires the detection of neutrons in the energy range between $\mathrm{E}_{\mathrm{n}} \approx 1$ and $3 \mathrm{MeV}$ with high efficiency. Most of the direct experiments performed so far in laboratories at Earth's surface using different detection techniques were limited by neutron background arising from cosmic-ray induced reactions, by radio-impurities in the detection setup and by cosmic-ray induced high energy $\gamma$-ray background. Recently, the reaction was also indirectly measured with the Trojan Horse Method [26]. For direct measurements, significant progress can only be achieved if the background is successfully reduced, as can be done in a deep underground laboratory as LNGS.

The ${ }^{22} \mathrm{Ne}(\alpha, n)^{25} \mathrm{Mg}$ is the dominant neutron source responsible for the weak component of the sprocess in the massive stars, both during core helium burning and in shell carbon burning. Moreover, the ${ }^{22} \mathrm{Ne}(\alpha, \mathrm{n})^{25} \mathrm{Mg}$ also affects the abundance of ${ }^{60} \mathrm{Fe}$ ejected during type II supernova explosion and the peak luminosity of type Ia supernovae [27]. For more than thirty years, direct measurements of the ${ }^{22} \mathrm{Ne}(\alpha, n)^{25} \mathrm{Mg}$ reaction have been performed close to the energy region of interest to the s-process: $\mathrm{E}_{\mathrm{cm}}=600 \pm 300 \mathrm{keV}$ but the resonance strengths from the different measurements disagree by up to a factor of 5 [27], a value much larger than the quoted uncertainties. The lowest energy resonance measured is located at $\mathrm{E}_{\alpha}=831 \mathrm{keV}$, just at the upper edge of the energy region of interest. Studies of the relative resonance contributions to the reaction rate show that, at the relevant temperatures to the s-process, the resonance at $\mathrm{E}_{\alpha}=831 \mathrm{keV}$ and those at lower energies are the most important.

Also in this case, previous direct measurements of the ${ }^{22} \mathrm{Ne}(\alpha, n)^{25} \mathrm{Mg}$ reaction were mainly hampered by extremely low count rates and by natural background due to cosmic rays. The direct underground measurement, taking advantage of the background reduction and of improved set-up for neutron detection, possibly including also the measurement of neutrons' energy, will significantly improve the knowledge of this reaction. In particular, a precise measurement of the known resonance down to the one at $\mathrm{E}_{\alpha}=831 \mathrm{keV}$ will be performed at first, followed then by a detailed search for unknown resonances down to $\mathrm{E}_{\alpha} \sim 600 \mathrm{keV}$.

After the submission of the LoI, an intense interaction took place between the LUNA collaboration on one side and the LNGS Scientific Committee and the LNGS management on the other side. Two more documents (LoI addendum [28] and LoI update [29]) were submitted by the LUNA collaboration to the LNGS Scientific Committee. A dedicated committee of scientists covering 
different expertise in the field of nuclear astrophysics was established. This produced a very positive report on the LUNA MV project, strongly recommending its realization at LNGS. The so-called "B node" at LNGS was identified as the best possible place to install the accelerator underground, due to its size and distance from the other LNGS experiments. The so called "LUNA-MV project" startedup: it foresees the realization of a worldwide unique facility inside the underground Gran Sasso Laboratory centred on a 3.5 MV single-ended accelerator for hydrogen and helium. Two different beam lines are foreseen devoted to solid and gas target experiments, respectively. This will allow us to study the key reactions of Helium burning described above and to re-investigate the ${ }^{3} \mathrm{He}\left({ }^{4} \mathrm{He}, \gamma\right){ }^{7} \mathrm{Be}$ reaction over a wide energy range in order to further diminish its experimental uncertainty. The $\mathrm{B}$ node site needs a complete refurbishment: the sealing and water proofing of the floor, the construction of an experimental hall, the electrical and safety plants, etc. A special project ("Progetto premiale") has been submitted to the Italian Research Ministry, after approval by INFN management, with a total request of 6,4 millions of euros in five years, 2.805 of which for the year 2012. The money for the year 2012 has been granted and will allow us to completely refurbish the node B site and buy the core of the 3.5 MV accelerator. To complete the facility with the beam lines, shielding and detectors approximately 3 more millions are requested. The funding of the first year of the "Progetto Premiale"' has given an exceptional push to the LUNA MV project with respect to the preparation of all the technical documents and executive projects necessary for the tendering procedures, both for site preparation and for the machine. A key issue here is the realization of a neutron shielding to the rest of the laboratory and to the internal rock "walls" to preserve the "low background" characteristic of LNGS. In order to evaluate the neutron fluxes potentially produced by the reactions above described (note that also the ${ }^{12} \mathrm{C}(\alpha, \gamma){ }^{16} \mathrm{O}$ reaction is producing a neutron background due to the parasitic ${ }^{13} \mathrm{C}(\alpha, n){ }^{16} \mathrm{O}$ component given by ${ }^{13} \mathrm{C}$ impurities in the ${ }^{12} \mathrm{C}$ solid target), a series of GEANT4 simulations have been developed, assuming a worst-case scenario of $2000 \mathrm{n} / \mathrm{s}$ of $5.6 \mathrm{MeV}$ energy (the maximum possible energy foreseen). According to the simulations, an effective shielding can be achieved by isolating the LUNA MV experimental area from the rest of the laboratory by two labyrinths composed of thick walls covered by a "roof". The walls will be composed of a sandwich of HDPE $(5 \% \mathrm{~B})$, concrete and borated concrete. Moreover, a $10 \mathrm{~cm}$ HDPE (5\% B) cover of the rock "walls" and a $20 \mathrm{~cm}$ concrete coverage of the pavement will complete the shielding. The results of the GEANT4 simulations are very encouraging since the neutron fluxes just outside the shielding are less than $1 \%$ of the natural LNGS flux.

The LUNA collaboration has also organized a workshop at LNGS in February 2013 in order to start-up the new LUNA-MV collaboration. About 60 people were present coming mainly from Europe but also Asia and United States of America. As a final outcome, adhesions to the project have been collected and the constitution of working groups on specific scientific/technical issues related to the LUNA MV project, such as the neutron detector for the study of ${ }^{13} \mathrm{C}(\alpha, n){ }^{16} \mathrm{O}$ and ${ }^{22} \mathrm{Ne}(\alpha, \mathrm{n}){ }^{25} \mathrm{Mg}$ reactions, started. Soon involved people will be called also for the drafting of the official Memorandum of Understanding where the agreements among different financing agencies will also be included.

\section{References}

1. C. Rolfs and W. Rodney, Cauldrons in the Cosmos (University of Chicago Press, Chicago, 1988 ) ; C. Iliadis, Nuclear Physics of Stars (WILEY-WCH, Verlag, 2007)

2. U. Greife et al., NIM A 350, 327 (1994)

3. A. Formicola et al., NIM A 507, 609 (2003)

4. R. Bonetti et al., PRL 82, 5202 (1999)

5. C. Casella et al., NPA 706, 203 (2002)

6. H. Costantini et al., PLB 482, 43 (2000)

7. A. Formicola et al., Phys. Lett. B 591, 61 (2004)

8. G. Imbriani et al., Eur. Phys. J. A 25, 455 (2005) 
9. A. Lemut et al., Phys. Lett. B 634, 483 (2006)

10. G. Imbriani et al., Astron. Astrophys. 420, 625 (2004)

11. D. Bemmerer et al., Phys. Rev. Lett. 97, 122502 (2006)

12. F. Confortola et al., Phys. Rev. C 75065803 (2007)

13. H. Costantini, et al., Nucl. Phys. A 814, 144 (2008)

14. E. Adelberger et al., Rev. Mod. Phys 83, 195 (2011)

15. A. Serenelli et al., Astroph. J, 743, 24 (2011)

16. A. Caciolli et al., Astron. Astrophys. 533, A66 (2011)

17. D. Scott et al., Phys. Rev. Lett 109, 202501 (2012)

18. B. Limata et al., Phys. Rev. C 82, 015801 (2010)

19. F. Strieder et al., Phys. Lett. B 707, 60 (2012)

20. O. Straniero et al., Astroph. J, 763, 100 (2013)

21. H. Costantini et al., Rep. on Prog. in Phys. 72, 086301 (2009)

22. C. Broggini et al., Ann. Rev. of Nucl. and Part. Sci. 60, 53 (2010)

23. The LUNA Collaboration, LUNA-MV Letter of Intent- LoI 42/07

24. C. Tur et al., Astroph. J. 718, 357 (2010)

25. E. M .Burbidge et al., Rev. Mod. Phys. 29547 (1957)

26. M. La Cognata et al., Phys. Rev. Lett. 109232701 (2012)

27. R. Longland et al., Phys. Rev. C 85065809 (2012)

28. The LUNA Collaboration, Addendum to the LUNA-MV Letter of Intent- LoI 42/07

29. A. Guglielmetti, "Update on the LUNA MV Letter of Intent 42/07”, February $26^{\text {th }}, 2010$ 